\title{
A Influência do Timbre na Aquisição de Habilidades Musicais Rudimentares
} The Influence of Timbre in the Acquisition of Rudimentary Musical Skills La Influencia del Timbre en la Adquisición de Habilidades Musicales Rudimentarias

\author{
Raone Mateus Rodrigues ${ }^{1}$ \\ Átila Moreira Cedro \\ Rebeca Mendes Fonseca \\ Clarisse Vasconcelos Friedlaender \\ Pedro Henrique Ribeiro Torres \\ Vinicius Figueiredo de Oliveira \\ Pedro Henrique Guimarães Couto \\ Universidade Federal de Minas Gerais (UFMG) \\ Antonio Carlos Leme Junior \\ Universidade Federal de São Carlos (UFSCAR) \\ Edson Massayuki Huziwara \\ Universidade Federal de Minas Gerais (UFMG)
}

\begin{abstract}
Resumo
O objetivo do experimento foi investigar os efeitos de diferentes timbres no processo de aprendizagem de discriminações de acordes musicais. Dezessete estudantes universitários participaram. O procedimento consistiu em ensinar relações condicionais entre quatro diferentes acordes e suas representações pictóricas (i.e., X, Xm, Xm-5e X+5). Para nove participantes, os acordes foram tocados por um piano (Grupo-piano) e, para outros oito participantes, os mesmos acordes foram tocados por um violão (Grupo-violão). Em ambos os grupos, os participantes aumentaram a sua porcentagem de respostas corretas ao longo do procedimento de ensino. A análise dos dados também sugere que os participantes do Grupo-violão apresentaram maiores percentuais de acerto quando comparados com os participantes do Grupo-piano. Trabalhos recentes que utilizaram acordes em tarefas de discriminação condicional desconsideraram questões relacionadas ao timbre e nossos resultados sugerem que essa variável poderia modular o processo de aprendizagem e deve ser considerada em futuros estudos sobre o tema.

Palavras-chave: timbre, discriminação condicional, acordes musicais, estudantes universitários
\end{abstract}

\begin{abstract}
The present experiment aimed to investigate the effects of different timbre in the learning process of musical chord discriminations. Seventeen college students participated. The procedure was developed for teaching conditional relations among four different musical chords and its pictorial representations (i.e., $X, X m, X m-5 e X+5)$. Nine participants were given a teaching procedure in which musical chords were played by a piano (Piano Group) and other eight participants were given a teaching procedure in which the same musical chords were played by an acoustic guitar (Guitar Group). In both groups, participants increased their percentage of correct responses throughout the teaching procedure. Beyond that, data analysis also suggests the Guitar Group participants showed higher percentage of correct responses when compared to the Piano Group participants. The most recent works that used musical chords in conditional discrimination tasks did not consider issues related to the timbre. Nonetheless, our findings suggest that this variable could modulate the learning process and must be considered in futures studies about this matter.
\end{abstract}

Keywords: timbre, conditional discrimination, musical chords, college students

\section{Resumen}

Este experimento tuvo como objetivo investigar los efectos de diferentes timbres en el proceso de aprendizaje de discriminaciones de acordes musicales. Diecisiete estudiantes universitarios participaron. El procedimiento fue desarrollado para la enseñanza de relaciones condicionales entre cuatro diferentes acordes musicales y sus representaciones pictóricas (i.e., $\mathrm{X}, \mathrm{Xm}, \mathrm{Xm}-5 \mathrm{e} \mathrm{X}+5$ ). Nueve participantes recibieron

${ }^{1}$ Endereço de contato: Rua Benedito José das Neves, n. 451, Bairro São Gonçalo, Contagem, Minas Gerais. CEP: 32042-000. E-mail: raonemateusrodrigues@gmail.com 
un procedimiento de enseñanza en el cual los acordes musicales fueron tocados por un piano (Grupopiano) y otros ocho participantes recibieron un procedimiento de enseñanza en que los mismos acordes musicales fueron tocados por una guitarra (Grupo-guitarra). En ambos grupos los participantes aumentaron su porcentaje de respuestas correctas a lo largo del procedimiento de enseñanza. Además, el análisis de los datos también sugiere que los participantes del Grupo-guitarra presentaron más porcentajes de aciertos que los participantes del Grupo-piano. Trabajos más recientes que utilizaron acordes musicales en tareas de discriminación condicional no consideraron cuestiones relacionadas con el timbre. Sin embargo, nuestros resultados sugieren que esa variable podría modular el proceso de aprendizaje y debe de ser considerada en futuros estudios sobre el tema.

Palabras clave: timbre, discriminación condicional, acordes musicales, estudiantes universitarios

O convívio em sociedade exige que sejamos capazes de interpretar vários sistemas simbólicos. Símbolos estão presentes, por exemplo, quando conversamos; quando lemos ou escrevemos um texto; quando categorizamos e/ou mensuramos o mundo utilizando números; e em muitas outras situações cotidianas. Na Análise do Comportamento, utilizando um paradigma que descreve a formação e a manutenção de classes de estímulos equivalentes, a aquisição da função simbólica é estudada experimentalmente a partir do estabelecimento de relações condicionais e arbitrárias entre estímulos (de Rose, 1993; Sidman, 1971; 1994; 2000; Sidman \& Tailby, 1982).

Tradicionalmente, a formação de classes de estímulos equivalentes tem sido investigada utilizando o procedimento de emparelhamento de acordo com o modelo (matching to sample). Nesse procedimento, participantes são requisitados a escolher um dentre dois (ou mais) estímulos, chamados estímulos de comparação (e.g., B1 e B2). A cada tentativa, a escolha de um determinado estímulo de comparação será reforçada ou não a depender de uma relação condicional como estímulo modelo apresentado. Mais especificamente, em tentativas que apresentarem o estímulo A1 como modelo, a escolha do comparação B1 será reforçada, enquanto que a escolha de B2 não produzirá consequências reforçadoras. De maneira análoga, em tentativas que apresentarem o estímulo A2 como modelo, a escolha do comparação B2 será reforçada, enquanto que a escolha de B1 não produzirá consequências reforçadoras.

Um considerável conjunto de dados experimentais têm demonstrado que indivíduos treinados a relacionar condicionalmente estímulos perceptual ou fisicamente distintos, em procedimentos como os descritos anteriormente, podem estabelecer novas relações condicionais entre esses mesmos estímulos sem qualquer treino adicional (Bataglini, Almeida-Verdu, \& Bevilacqua, 2013; Bortoloti \& de Rose, 2012; de Rose, de Souza, \& Hanna, 1996; de Souza \& de Rose, 2006; Sidman, 1971; 1994; Sidman \& Tailby, 1982; Wilkinson \& Mcllvane, 2001). Essas novas relações, também chamadas de relações emergentes, têm sido explicadas pelo fato dos estímulos envolvidos terem se tornado substituíveis no controle de alguns padrões comportamentais, formando as chamadas classes de estímulos equivalentes (Sidman, 1994).

Em uma classe de equivalência, todos os estímulos relacionam-se entre si segundo propriedades reflexivas, simétricas e transitivas, mesmo que essas propriedades relacionais não tenham sido diretamente ensinadas (Sidman \& Tailby, 1982). De modo geral, em estudos sobre equivalência de estímulos, ensina-se apenas um conjunto restrito de relações condicionais entre estímulos (e.g., $\mathrm{AB}$ e BC) e, em uma situação posterior, na ausência de qualquer reforçamento programado, avalia-se se o treino prévio de fato produziu a emergência dos repertórios que seriam coerentes com a formação de classes de equivalência (Sidman, 1994). 
A propriedade de reflexividade é avaliada por meio de tentativas nas quais espera-se que o participante relacione cada estímulo das relações condicionais treinadas com outro idêntico a ele mesmo (relações AA, BB e CC). A propriedade de simetria é avaliada em tentativas nas quais são invertidas as posições dos estímulos de uma relação modelo-comparação previamente reforçada (relações BA e CB). A transitividade é verificada apresentando-se como modelo e comparação, em uma mesma tentativa, dois estímulos que foram previamente relacionados a um terceiro estímulo comum (relação $A C$ ).

Desde o estudo pioneiro conduzido por Sidman (1971), grande parte dos experimentos com participantes humanos acerca do paradigma de equivalência de estímulos investigaram processos envolvidos na aquisição de repertórios de leitura e escrita em populações com as mais variadas características (e.g., de Rose, de Souza, \& Hanna, 1996; de Souza \& de Rose, 2006; de Souza, de Rose, \& Domeniconi, 2009; Serejo, Hanna, de Souza, \& de Rose, 2007; Reis, de Souza, \& de Rose, 2009). A partir de uma análise dos estímulos utilizados nesses experimentos, torna-se possível dimensionar o trabalho cuidadoso e criterioso no momento de escolhê-los e/ou construí-los. Para além do cuidado experimental, tal trabalho justifica-se ao considerar que a utilização de estímulos adequados aumenta significativamente as possibilidades de aprendizagem dos indivíduos que são submetidos a esses procedimentos, ou seja, a escolha de estímulos adequados pode modular de forma determinante os resultados obtidos pelos participantes que realizam o procedimento de ensino.

Pesquisas sobre a formação de classes de equivalência têm se mostrado uma via próspera não apenas para o ensino de habilidades de leitura e escrita, mas também em uma grande variedade de situações. Apoiadas no paradigma da equivalência de estímulos estão inúmeras pesquisas que têm se dedicado a ensinar habilidades matemáticas avançadas, leitura em braile e, também, habilidades musicais (Fiorentini, Arismendi, \& Yorio, 2012, para revisão detalhada). Especificamente sobre o ensino de habilidades musicais, várias são as pesquisas que descreveram o comportamento de produzir/ouvir música a partir do ensino de relações condicionais entre estímulos auditivos e visuais (e.g., Acín, Garcia, Zayas, \& Dominguez, 2006; Batitucci, 2007; Filgueiras, 2011; Hayes, Thompson, \& Hayes, 1989; Huber, 2010; Madeira, 2012; Pereira, 2012; Perez \& de Rose, 2010; Reis, 2007; Tommys \& Fazey, 1999). Madeira (2012), por exemplo, conduziu um experimento em que 11 estudantes dos ensinos fundamental e médio foram ensinados a relacionar condicionalmente notas musicais $(A)$ e notações na partitura (B); e, também, as notas musicais (A) e os nomes impressos das notas musicais (C). A análise dos resultados apontou que 10 participantes obtiveram altos percentuais de acertos nos testes das relações entre notações na partitura e nomes impressos das notas (testes $B C$ ), e nas relações entre nomes impressos das notas e notações na partitura (testes $C B$ ). No estudo de Pereira (2012) foram ensinadas relações condicionais entre o som de três notas musicais (A) e as notações referentes na partitura (B); e relações condicionais entre o som dessas mesmas três notas musicais (A) e as teclas correspondentes no piano (C). A análise dos resultados indicou que cinco dos seis participantes atingiram $100 \%$ de acertos nos testes para verificar a emergência de relações condicionais entre as notações na partitura e as figuras dos teclados (teste BC) e entre as figuras de teclados e as notações na partitura (teste CB).

Apesar dos resultados positivos obtidos nos experimentos que relacionaram equivalência de estímulos e ensino de habilidades musicais (Acín et al., 2006; Batitucci, 2007; Filgueiras, 2011; Hayes et al., 1989; Huber, 2010; Madeira, 2012; Pereira, 2012; Perez \& de Rose, 2010; 
Reis, 2007; Tommys \& Fazey, 1999), talvez fosse necessário um estudo sistemático das características dos estímulos musicais geralmente utilizados nesses experimentos. Resultados advindos desses estudos seriam úteis para determinar, por exemplo, quais características devem ser observadas na seleção dos estímulos musicais a serem utilizados. Ao tomar como exemplo o ensino de habilidades de leitura e escrita, podemos observar que vários critérios são utilizados para a escolha dos estímulos que compõem tais procedimentos. Por exemplo, utilizar palavras do tipo "consoante-vogal", como FIGO ou GALO, evitar o uso de palavras que possuam dígrafos, como ABELHA ou GALINHA, ou ainda, evitar palavras com som anasalado, como TUCANO (de Morais, 2013; de Souza et al., 2009; Reis et al., 2009; Serejo et al., 2007).

Tais critérios não são claramente definidos para a escolha dos estímulos musicais. A maioria dos experimentos citados (e.g., Acín et al., 2006; Batitucci, 2007; Filgueiras, 2011; Hayes et al., 1989; Madeira, 2012; Pereira, 2012; Perez \& de Rose, 2010; Reis, 2007; Tommys \& Fazey, 1999), por exemplo, utilizou notas ou acordes musicais tocados em piano. No entanto não parece haver qualquer tipo de avaliação empírica com relação à discriminabilidade desses sons quando comparados a de outros instrumentos musicais. O estudo sobre procedimentos para ensino de habilidades musicais e, principalmente, esforços no sentido potencializar a eficácia dos procedimentos de ensino justificam-se na medida em que pesquisas recentes sugerem que o ensino de tais habilidades tem impacto direto na promoção da saúde e no aprimoramento de habilidades cognitivas tanto em idosos (Bugos, Perlstein, McCrae, Brophy, \& Bedenbaugh, 2007; Seinfeld, Figueroa, Ortiz-Gil, \& Sanchez-Vives, 2013; Verghese et al., 2003) quanto em crianças e jovens (Hallam, 2010; Schellenberg, 2004).

O presente experimento pretendeu contribuir exatamente com a avaliação da discriminabilidade de estímulos musicais apresentados por diferentes instrumentos. Mais especificamente, o objetivo consistiu em comparar o processo de aquisição de relações condicionais em tarefas de MTS tendo sons de acordes como modelos e palavras impressas como comparações. Para um grupo de participantes, os acordes foram tocados em piano. Para um segundo grupo de participantes, os acordes foram tocados no violão. Em grande medida, as diferenças entre acordes tocados por diferentes instrumentos estão relacionadas às diferenças da característica sonora denominada timbre.

Uma conceituação recorrente no meio musical aborda o timbre como a cor ou a qualidade do som, sendo aquilo que permite ao ser humano distinguir entre diferentes instrumentos e vozes. Uma conceituação psicoacústica define o timbre como uma qualidade elementar do som, próxima da altura e da intensidade. Contudo, até certo ponto, essa qualidade se situa fora das noções que descrevem altura (i.e., frequência) e a intensidade do som. De forma mais específica, o timbre é definido como um atributo do som que é multidimensional. Nessa perspectiva, questões recorrentes em pesquisas que abordam o timbre são: como determinar quais são essas dimensões, como mensurar essas dimensões e a correlação entre elas (Caclin, McAdams, Smith, \& Winsberg, 2005; Grey, 1977; Halpern, Zatorre, Boufford, \& Johnson, 2004; Marozeau, Cheveigne, McAdams, \& Winsberg, 2003; McAdams, Winsberg, Donnadieu, de Soete, \& Krimphoff, 1995; Wessel, 1979). Em termos técnicos, o timbre é a forma de onda da vibração sonora (Fernandes, 2002), ou seja, dois instrumentos distintos (e.g., violão e piano) tocando a mesma nota, na mesma intensidade e com a mesma duração terão, na sua análise espectral, duas formas de ondas distintas que permitirão identificar a origem sonora. 


\section{Método}

\section{Participantes}

Participaram do estudo 17 estudantes universitários, sendo 12 mulheres e cinco homens, com idade entre 18 e 30 anos e sem problemas auditivos aparentes. Todos os participantes tiveram acesso e assinaram o Termo de Consentimento Livre e Esclarecido antes de iniciar o procedimento.

\section{Local e Equipamentos}

A coleta de dados foi realizada em uma sala de $2 \mathrm{~m} \times 3 \mathrm{~m}$, com boa iluminação e com baixo nível de ruído. As tarefas experimentais foram programadas utilizando-se o software MTS (Dube, 1991), instalado em dois computadores com processador Intel ${ }^{\circledR} 173770$, sistema operacional Windows Vista ${ }^{\circledR}$ e com tela de 23,6".

Para a gravação dos estímulos auditivos foram utilizados um teclado modelo CSR 2177, um violão da marca Eagle ${ }^{\circledR}$ modelo $\mathrm{CH}-70 \mathrm{~F}$ RB e um piano virtual gravado no software GarageBand $^{\circledR}$. Para a apresentação desses estímulos auditivos foi utilizado um fone de ouvido modelo Coby ${ }^{\circledR} \mathrm{CVH}$ 145, com impedância de 32 Ohms, amplitude de frequência entre 20 e $20.000 \mathrm{~Hz}$, sensibilidade de $106 \mathrm{~dB}$ e potência de entrada de $15 \mathrm{~mW}$.

\section{Estímulos Visuais e Auditivos}

Durante o Teste Inicial, os estímulos visuais utilizados foram as palavras impressas "MAIOR" e "MENOR" escritos em fonte Times New Roman, tamanho 100 e cor vermelha (i.e., R 255, $\mathrm{G} 0$ e B 0). Os estímulos auditivos utilizados foram acordes tocados em um teclado. Cada estímulo auditivo tinha duração de 3 segundos.

Durante a etapa de Treino Discriminativo, por sua vez, foram utilizados os símbolos impressos $\mathrm{X}, \mathrm{Xm}, \mathrm{Xm}^{-5} \mathrm{e} \mathrm{X}^{+5}$. Esses símbolos substituem a tradicional cifragem dos acordes $\mathrm{C}$, $\mathrm{Cm}, \mathrm{Cm}^{-5} \mathrm{e}^{+5}$ e foram escolhidos porque o foco do presente estudo era a identificação do tipo dos acordes (e.g., acorde maior e menor) e não a nomeação do tipo mais a nota fundamental (e.g., acorde de dó maior, ré menor, etc.). Os estímulos visuais também foram escritos em fonte Times New Roman, tamanho 100 e cor vermelha (i.e., R 255, G 0 e B 0). Como explicitado pela própria denominação dada aos diferentes grupos, os participantes designados para o Grupo-violão foram submetidos a um treino em que os estímulos auditivos foram acordes tocados em um violão. Da mesma forma, os participantes designados para o Grupopiano foram submetidos a um treino em que os estímulos auditivos foram acordes tocados em um piano.

A Tabela 1 apresenta as frequências dos estímulos auditivos utilizados em cada uma das etapas do experimento. Por exemplo, o acorde de Db é constituído pelas notas ré bemol (138,59 Hz), fá $(174,61 \mathrm{~Hz})$ e lá bemol $(207,65 \mathrm{~Hz})$ e o acorde Dbm é constituído pelas notas ré bemol $(138,59 \mathrm{~Hz})$, fá bemol $(164,81 \mathrm{~Hz})$ e lá bemol $(207,65 \mathrm{~Hz})$. A única diferença entre ambos os acordes é a segunda nota de sua composição, ou seja, as notas fá e fá bemol, nos acordes Db e Dbm, respectivamente. Importante destacar que, para a apresentação dos estímulos auditivos, as notas que compunham cada acorde eram tocadas de forma simultânea. 


\section{Tabela 1}

Estímulos auditivos utilizados em cada uma das etapas do procedimento

\begin{tabular}{|c|c|c|c|c|}
\hline $\begin{array}{c}\text { Etapa do } \\
\text { procedimento }\end{array}$ & Acorde & $\begin{array}{r}E \\
\text { Notas }\end{array}$ & $\begin{array}{l}\text { mulos Auditivos } \\
\text { nstituintes do acorde (f }\end{array}$ & quência) \\
\hline \multirow{4}{*}{ Pré-treino } & $\begin{array}{c}\mathrm{Db} \\
\mathrm{Dbm}\end{array}$ & Ré bemol $(138,59 \mathrm{~Hz})$ & $\begin{array}{c}\text { Fá }(174,61 \mathrm{~Hz}) \\
\text { Fá bemol }(164,81 \mathrm{~Hz})\end{array}$ & Lá bemol $(207,65$ Hz) \\
\hline & $\begin{array}{c}\mathrm{Eb} \\
\mathrm{Ebm}\end{array}$ & Mi bemol $(155,56 \mathrm{~Hz})$ & $\begin{array}{c}\text { Sol }(196,00 \mathrm{~Hz}) \\
\text { Sol bemol }(185,00 \mathrm{~Hz})\end{array}$ & Si bemol $(233,08 \mathrm{~Hz})$ \\
\hline & $\begin{array}{c}\mathrm{Ab} \\
\mathrm{Abm}\end{array}$ & Lá bemol $(207,65$ Hz) & $\begin{array}{c}\text { Dó }(261,63 \mathrm{~Hz}) \\
\text { Dó bemol }(246,94 \mathrm{~Hz})\end{array}$ & Mi bemol $(311,13 \mathrm{~Hz})$ \\
\hline & $\begin{array}{c}\mathrm{Bb} \\
\mathrm{Bbm}\end{array}$ & Si bemol $(233,08 \mathrm{~Hz})$ & $\begin{array}{c}\text { Ré }(293,66 \mathrm{~Hz}) \\
\text { Ré bemol }(277,18 \mathrm{~Hz})\end{array}$ & Fá $(349,23$ Hz) \\
\hline \multirow[b]{2}{*}{$\begin{array}{c}\text { Treino } \\
\text { Discriminativo }\end{array}$} & $\begin{array}{c}\mathrm{C} \\
\mathrm{Cm}\end{array}$ & \multirow{2}{*}{ Dó $(261,63 \mathrm{~Hz})$} & $\begin{array}{c}\text { Mi }(329,63 \mathrm{~Hz}) \\
\text { Mi bemol }(311,13 \mathrm{~Hz})\end{array}$ & Sol $(392,00 \mathrm{~Hz})$ \\
\hline & $\frac{\mathrm{C}^{+5}}{\mathrm{Cm}^{-5}}$ & & $\begin{array}{c}\text { Mi }(329,63 \mathrm{~Hz}) \\
\text { Mi bemol }(311,13 \mathrm{~Hz})\end{array}$ & $\begin{array}{c}\text { Sol sustenido }(415,30 \mathrm{~Hz}) \\
\text { Sol bemol }(369,99 \mathrm{~Hz})\end{array}$ \\
\hline
\end{tabular}

Os acordes maiores e menores foram utilizados por serem considerados consoantes. Esses acordes são considerados consoantes em razão de serem estáveis. Acordes dissonantes são assim considerados por gerarem tensão, tensão esta que é resolvida com a introdução de um acorde consoante. Outra característica dos acordes consoantes é que os intervalos entre as três notas que os compõem são frações simples e simétricas. Nessa perspectiva, um acorde maior tem um intervalo de quatro semitons entre a nota tônica e a terça maior, e um intervalo de três semitons entre a terça maior e a quinta justa. No caso de um acorde menor, existe um intervalo de três semitons entre a nota tônica e a terça menor, e um intervalo de quatro semitons entre a terça menor e a quinta justa. Tanto em acordes maiores quanto em menores, a quantidade de semitons entre a nota tônica e a quinta justa continua idêntico, ou seja, um intervalo de sete semitons em ambos os casos (Cardoso \& Mascarenhas, 1996; Sollberge, Rebe, \& Eckstein, 2003).

Os acordes maiores com quinta aumentada e menores com quinta diminuta foram utilizados para verificar se haveria variação na discriminabilidade entre diferentes tipos de acordes, de acordo com as unidades mínimas que os compõem. No caso de acordes maiores e menores, a única diferença entre eles está na segunda nota que os compõem. No acorde maior, a segunda nota é uma terça maior, no acorde menor a segunda nota é uma terça menor. Nos acordes maiores com quinta aumentada e nos acordes menores com quinta diminuta, a diferença entre eles está na segunda e terceira notas que os compõem. E o acorde maior com quinta aumentada tem a terça maior e a quinta aumentada, e acorde menor com quinta diminuta tem a terça menor e a quinta diminuta (Med, 1996).

Pesquisas relatam um grau de preferência a acordes consoantes quando comparados aos acordes dissonantes. Estudos demonstraram que participantes diante de acordes consoantes apresentaram mais respostas afetivas positivas, sendo que os mesmos participantes apresentaram mais respostas afetivas negativas diante de acordes dissonantes (e.g. Costa, Bitti, \& Bonfiglioli, 2000; Smith \& Williams, 1999; Sollberge, Rebe, \& Eckstein, 2003). 


\section{Procedimento}

O procedimento foi dividido em duas etapas, Teste Inicial e Treino Discriminativo. Em ambas as etapas, cada tentativa foi iniciada com a apresentação simultânea de um quadrado preto no centro da tela e um estímulo modelo sonoro (i.e., acorde). Após a apresentação do acorde, o participante deveria clicar no quadrado referido para sinalizar que havia ouvido o som (i.e., resposta de atenção). O som do acorde continuava a ser apresentado em intervalos regulares de 1 s enquanto a resposta de atenção não fosse emitida. A emissão da resposta de atenção produzia a apresentação dos estímulos de comparação nos vértices da tela. Foram apresentados dois e quatro estímulos de comparação durante o Teste Inicial e o Treino Discriminativo, respectivamente. A resposta do participante consistia em escolher, dentre as opções apresentadas, aquela que ele julgasse estar relacionada ao modelo.

\section{Teste Inicial}

O objetivo do Teste Inicial foi avaliar o repertório inicial dos participantes, garantindo que fossem selecionados para o Treino Discriminativo apenas aqueles que fossem incapazes de discriminar entre acordes maiores e menores. Essa etapa foi constituída por um bloco de 20 tentativas e era realizada por todos os participantes uma única vez, no início do experimento. Os estímulos modelos eram acordes maiores ou menores tocados no teclado e os estímulos de comparação eram as palavras impressas MAIOR e MENOR. Respostas consideradas corretas consistiam em escolher a palavra impressa MAIOR nas tentativas em que os acordes Db (ré bemol maior), Eb (mi bemol maior), Ab (lá bemol maior) e Bb (si bemol maior) fossem apresentados. Além disso, também foram consideradas respostas corretas escolher a palavra impressa MENOR nas tentativas em que os acordes Dbm (ré bemol menor), Ebm (mi bemol menor), Abm (lá bemol menor) e Bbm (si bemol menor) fossem apresentados. Importante salientar que, durante o Teste Inicial, as respostas de escolha dos participantes não foram consequenciadas. Todos os participantes que obtiveram percentual de acertos abaixo de $60 \%$ foram encaminhados para a etapa do Treino Discriminativo.

\section{Treino Discriminativo}

Nesta etapa, os participantes foram aleatoriamente alocados em dois grupos distintos. 0 primeiro grupo foi composto por oito participantes, e os estímulos modelo foram acordes tocados no violão (Grupo-violão). O segundo grupo, por sua vez, foi composto por nove participantes, e os estímulos modelo foram acordes tocados no piano (Grupo-piano). Importante salientar que os estímulos auditivos utilizados como modelos tinham o mesmo tempo de duração e estavam situados na mesma faixa de frequência (ver Tabela 1), diferenciando-se apenas com relação ao instrumento utilizado para a execução dos acordes e, consequentemente, no timbre dos estímulos auditivos apresentados.

O delineamento do treino foi exatamente igual para ambos os grupos, sendo composto por três blocos de treino com 32 tentativas cada um. Independente do instrumento utilizado para executar os acordes, cada bloco apresentava, em ordem semialeatória, oito tentativas com o acorde C (Dó maior), acrescidas de outras oito tentativas com o acorde Cm (Dó menor), mais oito tentativas com $\mathrm{C}^{5+}$ (Dó maior com $5^{\text {a }}$ aumentada) e, finalmente, oito tentativas 
com $\mathrm{Cm}^{-5}$ (Dó menor com $5^{\text {a }}$ diminuta). A única diferença entre os blocos estava relacionada à ordem de apresentação das tentativas.

Não foram utilizados critérios de aprendizagem durante o Treino Discriminativo. Os participantes de ambos os grupos passariam pelos três blocos de treino independentemente dos escores de acerto obtidos, e sua participação era encerrada após o terceiro bloco, sem qualquer relação com o desempenho apresentado.

Os estímulos de comparação foram desenhos esquemáticos que representavam a cifragem usual dada a esses acordes. Mais especificamente, utilizou-se o X para representar a cifragem de um acorde maior (e.g., C), utilizou-se também o Xm para representar a cifragem de um acorde menor (e.g., $\mathrm{Cm}$ ), o $\mathrm{X}^{+5}$ foi utilizado para representar a cifragem de um acorde maior com $5^{\text {a }}$ aumentada (e.g., $\mathrm{C}^{+5}$ ) e, por último, o $\mathrm{Xm}^{-5}$ foi utilizado para representar a cifragem de um acorde menor com $5^{\text {a }}$ diminuta (e.g., $\mathrm{Cm}^{-5}$ ). Sendo assim, em uma tentativa cujo modelo foi o som do acorde de Dó maior, o participante deveria escolher o estímulo de comparação X. Feito isso, seu responder era reforçado pela apresentação de uma série de estrelas na tela do computador, simultaneamente à apresentação do som de aplausos. Se o participante selecionasse qualquer um dos outros três estímulos de comparação, a tela escurecia por 1,5 s. A Figura 1 apresenta um desenho esquemático de uma tentativa do treino.

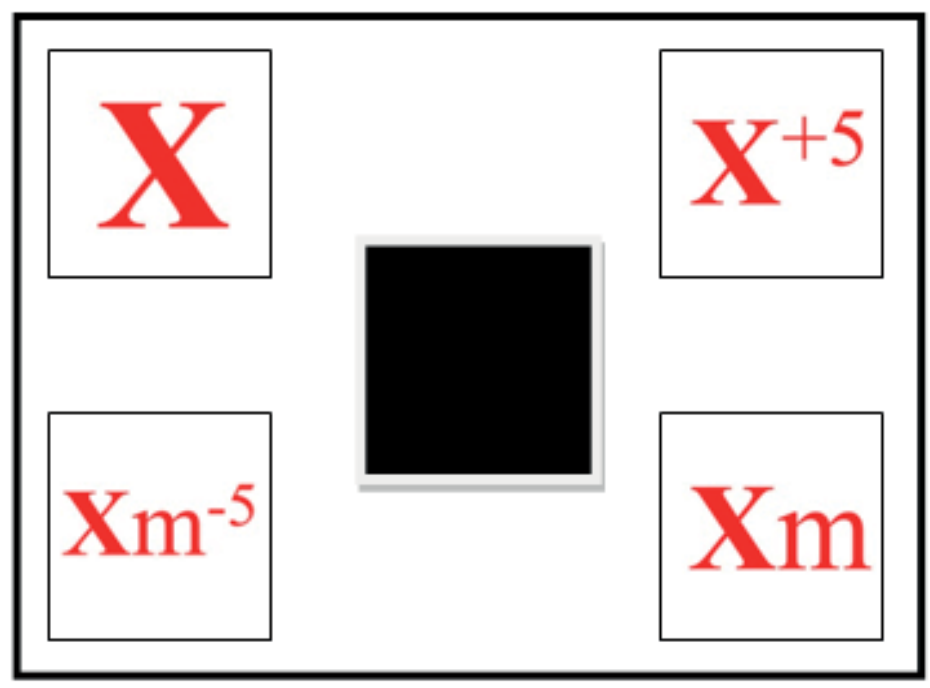

Figura 1. Desenho esquemático de uma tentativa da etapa de Treino Discriminativo.

As análises estatísticas do presente estudo foram feitas com o auxílio do teste de Student. Para as análises feitas dentro do mesmo grupo (i.e. Grupo-piano e Grupo-violão), foi utilizado o teste t pareado com o objetivo de comparar o percentual médio de acertos entre o primeiro e terceiro bloco do Treino Discriminativo. Para analisar as diferenças de desempenho entre o Grupo-violão e o Grupo-piano, foi realizado um teste t não pareado para cada um dos três blocos de treino. Considerou-se nível de significância de 5\%, e todas as análises foram realizadas no software Microsoft Excel (2013). 


\section{Resultados}

\section{Teste Inicial}

Os resultados do Teste Inicial são apresentados na Figura 2. Por meio da análise dessa figura é possível observar que ambos os grupos apresentaram percentual de acertos próximo ao nível do acaso (i.e., 50\%). O percentual médio de acertos dos participantes do Grupoviolão e do Grupo-piano foi, respectivamente, 50,6\% e 44,4\%. Esses valores sugerem que, no início do experimento, os participantes de ambos os grupos não eram capazes de, diante de um determinado acorde, selecionar a palavra impressa arbitrariamente designada como correta (i.e., MAIOR ou MENOR).

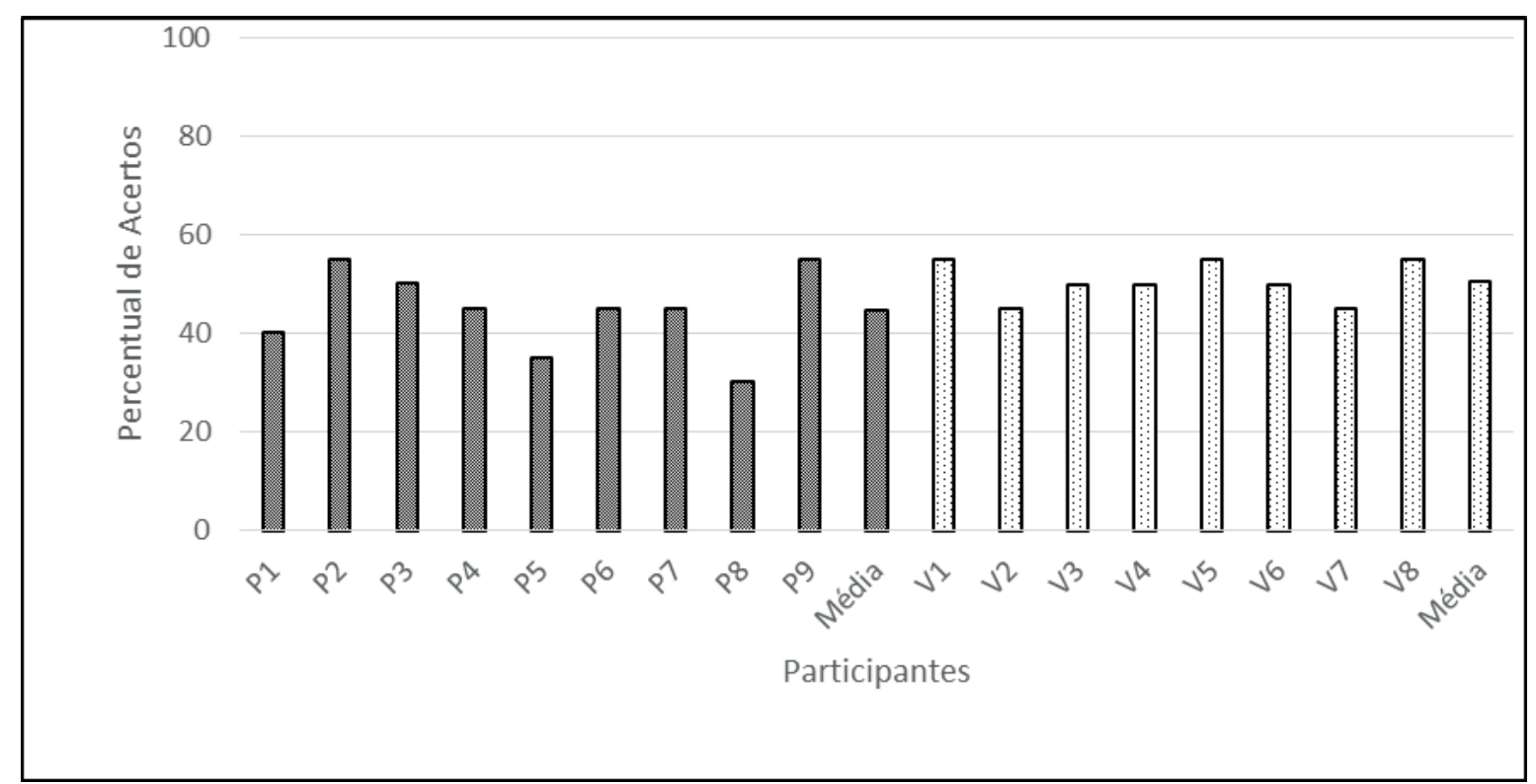

Figura 2. Percentual de acerto de todos os participantes na etapa de Pré-treino. Os valores de P1 à P9 representam os percentuais de acerto dos participantes do Grupo-piano e os valores de V1 a V8 representam os percentuais de acerto dos participantes do Grupo-violão.

\section{Treino Discriminativo}

Os resultados dos participantes do Grupo-piano obtidos no Treino Discriminativo são apresentados na Figura 3. É possível observar que, de maneira geral, o percentual de acerto de cinco dos nove participantes aumentou em cada bloco na medida em que o treino ocorreu. O participante P5 foi o que apresentou os menores escores de acertos, a saber, 22\%, $31 \%$ e $22 \%$ de acertos nos blocos 1, 2 e 3, respectivamente. O participante P9, por sua vez, foi aquele que obteve os maiores escores de acertos, 44\%, 66\% e 75\%, também respectivamente. O percentual médio de acerto de todos os participantes desse grupo foi de $30,6 \%$ no bloco 1, 44,4\% no bloco 2 e 45,5\% no bloco 3.

$\mathrm{O}$ resultado do teste $\mathrm{t}$ pareado (i.e., Grupo-piano: $\mathrm{p}=0.002$ ) indica que há diferença significativa no desempenho dos participantes desse grupo entre os blocos 1 e 3 do Treino Discriminativo. 


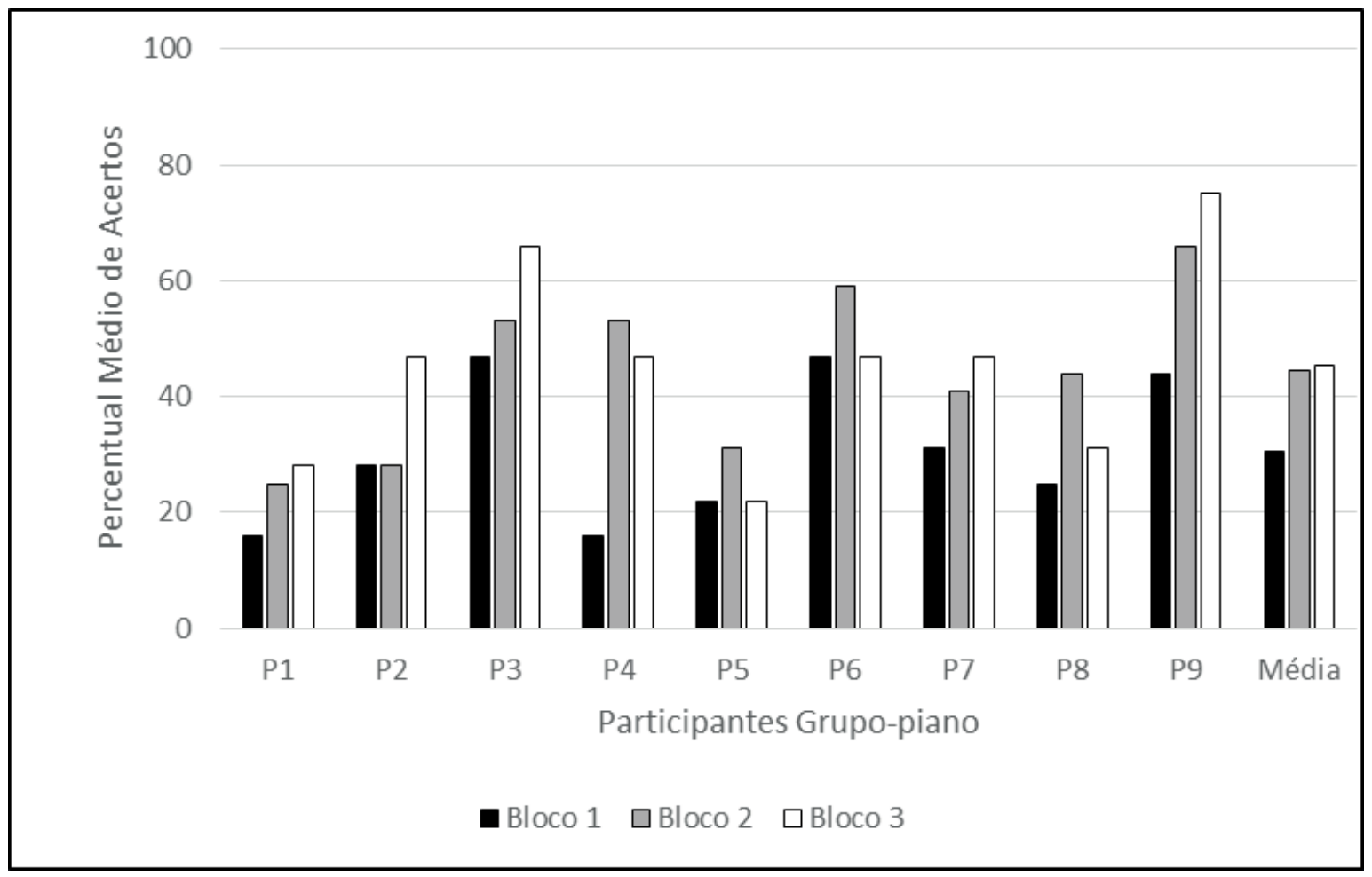

Figura 3. Percentual médio de acertos dos participantes do Grupo-piano por bloco de Treino discriminativo.

Os resultados dos participantes do Grupo-violão obtidos no Treino Discriminativo são apresentados na Figura 4. É possível observar que, de maneira semelhante ao que ocorreu com o Grupo-piano, para cinco dos oito participantes do Grupo-violão o percentual de acerto aumentou bloco após bloco. Apenas os participantes V1 e V5 obtiveram escores inferiores durante o terceiro bloco de treino, quando comparados ao primeiro e ao segundo blocos de treino. O participante $\mathrm{V} 4$ apresentou os maiores escores de acerto nessa etapa $(59,4 \%$, $87,5 \%$ e $87,5 \%$ nos blocos 1 , 2 e 3 , respectivamente), e o participante $V 5$, por sua vez, apresentou os menores percentuais de acerto $(34,4 \%, 34,4$ e $25 \%$ nos blocos 1 , 2 e 3 , respectivamente). O percentual médio de acerto de todos os participantes desse grupo foi de $40,6 \%$ no bloco $1,45,3 \%$ no bloco 2 e $55,8 \%$ no bloco 3 .

$\mathrm{O}$ resultado do teste $\mathrm{t}$ pareado (i.e., Grupo-violão: $\mathrm{p}=0.015$ ) indica que há diferença significativa no desempenho dos participantes desse grupo entre os blocos 1 e 3 do Treino Discriminativo. 


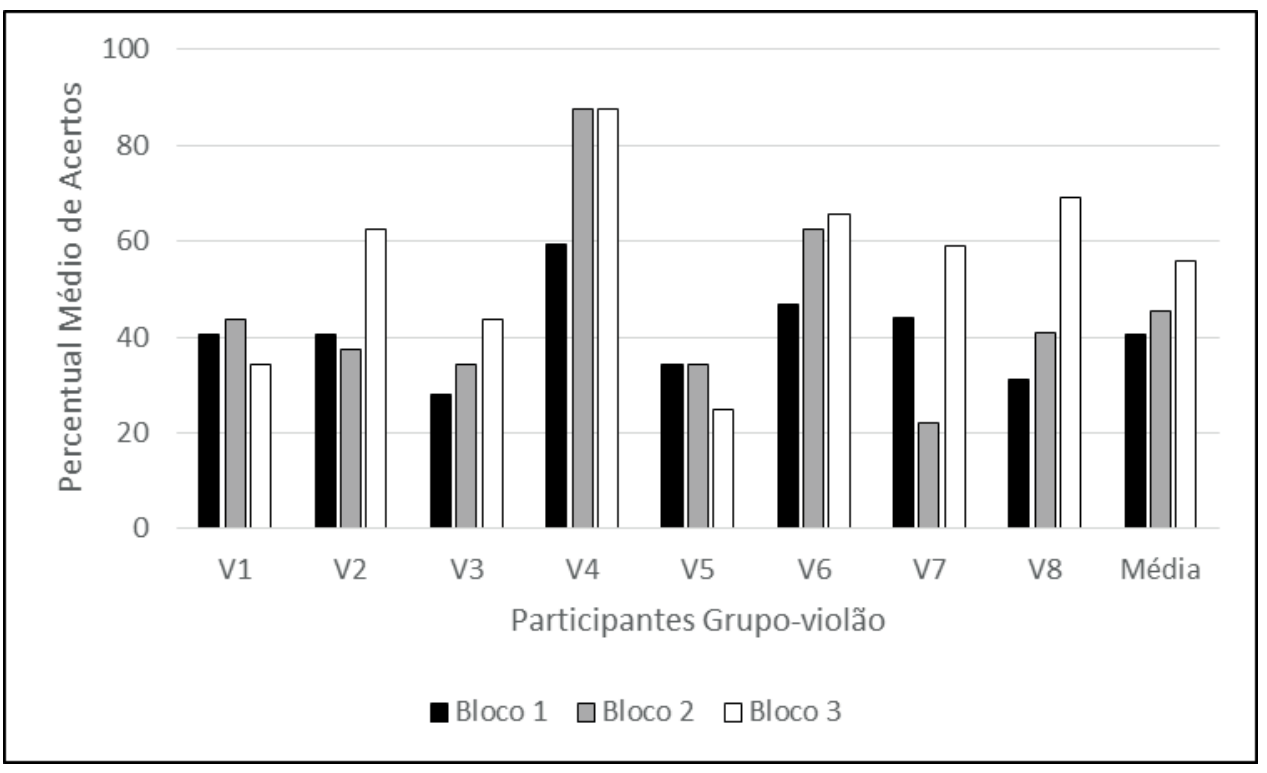

Figura 4. Percentual médio de acertos dos participantes do Grupo-violão por bloco de Treino discriminativo.

Por meio da análise das Figuras 3 e 4, é possível observar que a porcentagem média de acertos nos blocos do Treino Discriminativo foi superior para os participantes do Grupoviolão, quando comparados aos participantes do Grupo-piano. De acordo com a Figura 4, a porcentagem média de acertos por bloco do Grupo-violão foi de 40,6\%, 45,3\% e 55,8\% no primeiro, segundo e terceiro blocos de treino, respectivamente. A porcentagem média de acertos do Grupo-piano foi de 30,6\%, 44,4\% e 45,5\%, respectivamente (ver Figura 3). Comparando-se o desempenho dos participantes no primeiro e terceiro blocos de treino, é possível perceber que os participantes do Grupo-piano emitiram aproximadamente $10 \%$ a menos de respostas corretas quando comparados aos participantes do Grupo-violão. Apesar disso, o resultado do teste t não pareado indica que a diferença de desempenho entre os dois grupos só foi significativa para o primeiro bloco de Treino Discriminativo ( $p=0.044)$. Os resultados estatísticos para a comparação entre os blocos 2 e 3 são, respectivamente, $p=$ 0.458 e $p=0.141$.

A Figura 5 apresenta o número médio de acertos obtidos por cada tipo de acorde em cada um dos blocos de treino. Os participantes apresentaram uma tendência geral de aumento na quantidade de acertos ao longo dos blocos, fato que parece indicar a efetividade do procedimento para o ensino das relações condicionais pretendidas. É importante ressaltar que cada um dos acordes foi apresentado em oito oportunidades distintas e, portanto, a média dos participantes poderia variar entre zero e oito acertos. A análise dos resultados obtidos no bloco 1, por exemplo, indica que a média de acertos dos participantes do Grupo-violão foi de aproximadamente 4,6 das tentativas que apresentava $\mathrm{Cm}$ como modelo. Em contrapartida, o desempenho médio dos participantes do Grupo-piano para o mesmo $\mathrm{Cm}$ foi de 2,11 acertos. Além disso, os participantes do Grupo-violão obtiveram uma maior quantidade média 


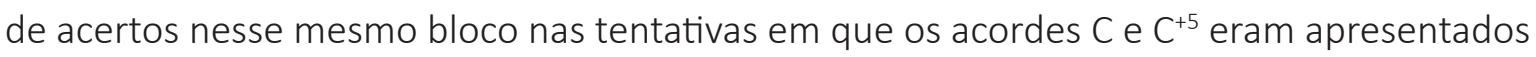
como modelo.

De forma similar ao ocorrido no bloco 1, os participantes do Grupo-violão obtiveram quantidade média de acertos superiores aos participantes do Grupo-piano na maioria das ocasiões em que tal comparação foi realizada. Apenas em duas, de um total de 12 ocasiões, é possível constatar desempenho superior dos participantes do Grupo-piano. Nomeadamente, no próprio bloco 1, nas tentativas em que $\mathrm{Cm}^{-5}$ foi apresentado como modelo, os participantes do Grupo-piano obtiveram 3,33 de média de acertos, enquanto que os participantes do Grupo-violão obtiveram 2,75 de média de acertos. No bloco 2, nas tentativas em que $C$ foi apresentado como modelo, a média de acertos do Grupo-piano foi de 3,67, enquanto o número médio de acertos do Grupo-violão foi de 2,13.

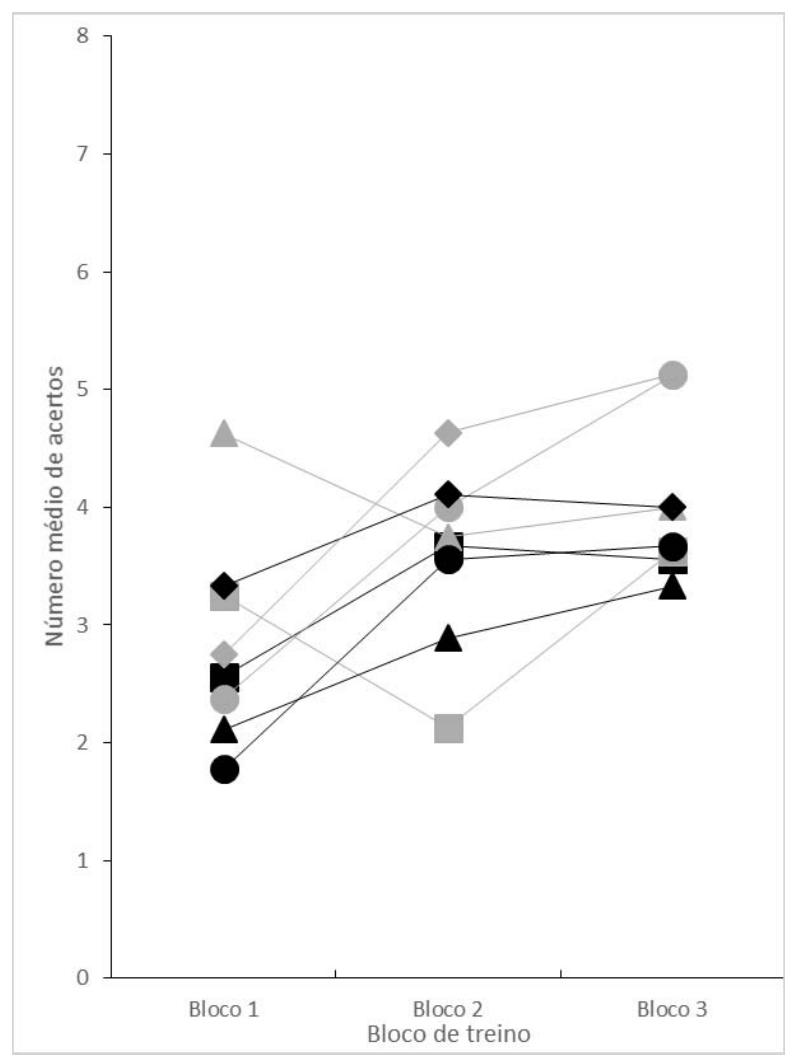

Figura 5. Quantidade média de acerto de cada acorde por bloco de treino. Os valores em preto representam os estímulos auditivos executados no piano (Grupo-piano) e os valores em cinza representam os estímulos auditivos executados no violão (Grupo-violão). As figuras geométricas representam cada um dos tipos de acorde, sendo que o triângulo representa o acorde de $\mathrm{Cm}$, o quadrado representa o acorde de C, o losango representa o acorde de $\mathrm{Cm}^{5}$, e, por último, o círculo representa o acorde de $\mathrm{C}^{+5}$.

\section{Discussão}

O objetivo do presente estudo foi verificar a influência do timbre na aprendizagem de habilidades musicais rudimentares. Tal aspecto ainda não foi amplamente estudado nas pesquisas que envolvem a análise experimental do comportamento e música (e.g., Acín et al., 2006; Batitucci, 2007; Filgueiras, 2011; Hayes et al., 1989; Huber, 2010; Madeira, 2012; 
Pereira, 2012; Perez \& de Rose, 2010; Reis, 2007; Tommys \& Fazey, 1999). A grande maioria desses estudos utiliza apenas o timbre do piano na confecção dos seus estímulos auditivos. Sabe-se que todos os estímulos auditivos possuem quatro características básicas: a altura (i.e., frequência das vibrações), a duração (i.e., a extensão de um determinado som), a intensidade (i.e., a amplitude das vibrações) e, por último, o timbre (i.e., a "cor" do som de determinado instrumento [Med, 1996]). Assim sendo, seria desejável que essas quatro características fossem estudadas de maneira sistemática em experimentos que utilizam estímulos musicais em suas tarefas. Os resultados advindos de tais estudos poderiam indicar os valores mais apropriados que cada uma dessas características deveria assumir para potencializar a aprendizagem das habilidades discriminativas envolvidas na percepção e produção musical.

No presente experimento, para verificar a influência exercida pelo timbre, todos os estímulos utilizados na etapa de Treino Discriminativo possuíam igual altura, duração e intensidade. O presente experimento surge num contexto em que cada vez mais se faz necessário o controle das propriedades dos estímulos utilizados nas tarefas experimentais, e é justamente por isso que a investigação a respeito da influência do timbre é relevante. Tal controle tem sido feito em inúmeras pesquisas que envolvem, por exemplo, a aquisição das habilidades de leitura e escrita (de Rose et al., 1996; de Souza \& de Rose, 2006; de Souza et al., 2009; Reis et al., 2009; Serejo et al., 2007).

Os resultados apresentados na seção anterior demonstraram que ambos os grupos experimentais melhoraram significativamente seu desempenho ao longo do treino, enquanto os percentuais médios de acertos no bloco 1 para o Grupo-violão e o Grupo-piano foram, respectivamente, $40,6 \%$ e $30,6 \%$, os percentuais médios de acertos no bloco 3 foram $55,8 \%$ e $45,5 \%$. Essas diferenças juntamente com os resultados estatisticamente significativos encontrados entre os blocos 1 e 3 de ambos os grupos indicam que a utilização do procedimento MTS se mostrou eficaz no ensino de relações condicionais envolvendo acordes musicais.

Apesar do aumento no porcentual de acerto ao longo dos blocos de treino, apenas um dos participantes conseguiu atingir escores superiores a $80 \%$ de acertos em um bloco (Participante 4 / Grupo-violão / blocos 2 e 3). Essa seria, portanto, uma evidência de que a quantidade de treino proposta não foi suficiente para que os participantes conseguissem adquirir a discriminação de acordes com suficiente maestria. Assim sendo, uma possível sugestão de estudo posterior seria planejar um procedimento em que se estipulasse um critério de aprendizagem (e.g., 90\% de acertos em dois blocos consecutivos) e que se medisse a quantidade média de sessões necessárias para obtê-lo. Caso o timbre do violão contribua mesmo para a aquisição das relações condicionais, os participantes do Grupo-violão alcançariam os critérios de aprendizagem mais rapidamente e, possivelmente, cometeriam menor quantidade de erros.

Também enquanto sugestão para futuros experimentos, seria importante considerar a possibilidade de um aumento considerável na quantidade de participantes em cada um dos grupos experimentais. A ausência de mais resultados com significância estatística podem, em alguma medida, ser explicados pelo reduzido número de participantes no presente experimento.

O conjunto dos resultados obtidos no Treino Discriminativo demonstra também que, por inspeção visual, os participantes do Grupo-violão obtiveram, em média, quantidades de acertos superiores aos dos participantes do Grupo-piano. Além disso, houve diferença estatística 
significativa $(p=0.044)$ entre ambos os grupos no bloco 1 indicando que o desempenho do Grupo-violão foi melhor do que o desempenho do Grupo-piano nesse bloco de Treino Discriminativo. Esses resultados parecem indicar que o timbre do instrumento pode interferir de maneira significativa na aquisição de relações condicionais quando acordes musicais são utilizados e que, portanto, tal fator deve ser levado em consideração ao se propor novos estudos envolvendo estímulos musicais. Ainda assim, quase a totalidade dos experimentos feitos anteriormente utilizou estímulos musicais executados por piano, ou seja, utilizaram um único timbre (e.g., Acín et al., 2006; Batitucci, 2007; Filgueiras, 2011; Hayes et al., 1989; Huber, 2010; Madeira, 2012; Pereira, 2012; Perez \& de Rose, 2010; Reis, 2007; Tommys \& Fazey, 1999) e esse fato pareceu não ocasionar problemas na aquisição das relações condicionais ensinadas. No entanto, de forma similar, quase a totalidade desses experimentos também foram conduzidos com estudantes universitários, ou seja, uma população bastante acostumada a ter contato com estímulos auditivos em forma de música e também a aprender e utilizar discriminações condicionais em suas atividades cotidianas.

Para permitir a utilização de procedimentos que envolvam ensino de habilidades musicais por meio de classes de equivalência para indivíduos com desenvolvimento atípico ou com restrições auditivas, como ocorre, por exemplo, para o ensino de habilidades de leitura e escrita ou matemática (de Rose et al., 1996; de Souza \& de Rose, 2006; de Souza et al., 2009; Reis et al., 2009; Serejo et al., 2007), seria importante investigar e identificar fatores e estratégias que facilitem a aquisição das relações condicionais. Se o timbre de um determinado instrumento influencia/facilita a aprendizagem por ser, talvez, mais facilmente discriminado, então esse fator deveria ser mais bem estudado e descrito. Ao considerar também que as evidências experimentais indicam que o ensino da música impacta na promoção da saúde e no aprimoramento de habilidades cognitivas em idosos e crianças (Bugos et al., 2007; Hallam, 2010; Schellenberg, 2004; Seinfeld et al., 2013; Verghese et al., 2003), procedimentos de ensino de habilidades musicais direcionados a essas populações parecem ser o caminho natural a ser trilhado nos próximos anos.

\section{Agradecimentos}

A realização do presente experimento contou com suporte financeiro do Instituto Nacional de Ciência e Tecnologia sobre Comportamento, Cognição e Ensino (INCT-ECCE; processo 465.686/2014-1). Gostaríamos de agradecer à Julia Borges e à Celline Cardoso Almeida Brasil pelo auxílio na realização dos testes estatísticos.

\section{Referências}

Acín, E. E., Garcia, A. G., Zayas, C. B., \& Dominguez, M. T. G. (2006). Formación de clases de equivalencia aplicadas al aprendizaje de las notas musicales. Psicothema, 18, 31-36.

Battaglini, M. P., Almeida-Verdu, A. C. M., \& Bevilacqua, M. C. (2013). Aprendizagem via exclusão e formação de classes de equivalência em crianças com deficiência auditiva e implante coclear. Acta Comportamentalia, 21, 20-35.

Batitucci, J. S. L. (2007). Paradigma da equivalência de estímulos no ensino de leitura desequências de notas musicais (Dissertação de Mestrado, Universidade de Brasília, Brasília, DF, Brasil). 
Bortoloti, R., \& de Rose, J. C. (2012). Equivalent stimuli are more strongly related after training with delayed matching than after simultaneous matching: a study using the implicit relational assessment procedure (IRAP). The Psychological Record, 62, 41-54.

Bugos, J. A., Perlstein, W. M., McCrae, C. S., Brophy, T. S., \& Bedenbaugh, P. H. (2007). Individualized piano instruction enhances executive functioning and working memory in older adults. AgingMentHealth, 11, 464-471.

Caclin, A., McAdams, S., Bennet, S. K., \& Winsberg, S. (2005). Acoustic correlates of timbre space dimensions: A confirmatory study using synthetic tones. Journal of Acoustical Association of America, 118(1), 471-482.

Cardoso, B., \& Mascarenhas, M. (1996). Curso completo de teoria musical e solfejo (14a ed., Vol. I). São Paulo: Irmãos Vitale.

Costa, M., Bitti, P. E. R., \& Bonfiglioli, L. (2000). Psychological connotations of harmonic musical intervals. Psychology of Music, 28, 4-22.

de Morais, M. A. S. (2013). Avaliação das vocalizações de crianças usuárias de implante coclear (Dissertação de Mestrado, Universidade Federal de Minas Gerais, Belo Horizonte, MG, Brasil).

de Rose, J. C. (1993). Classes de estímulos: Implicações para uma análise comportamental da cognição. Psicologia: Teoria e Pesquisa, 9, 283-303.

de Rose, J. C., de Souza, D. G., \& Hanna, E. S. (1996).Teaching reading and spelling: Exclusion and stimulus equivalence. Journal of Applied Behavior Analysis, 29, 451-469.

de Souza, D. G., de Rose, \& J. C., Domeniconi, C. (2009). Applying relational operants to reading and spelling. In R. A. Rehfeldt, \&Y. Barnes-Holmes (Eds.), Derived relational responding: Applications for learners with autism and other developmental disabilities (pp. 171-207). Oakland, Califórnia, EUA: New Harbinger Publications.

de Souza, D. G., \& de Rose, J. C. (2006). Desenvolvendo programas individualizados para o ensino de leitura. Acta Comportamentalia, 14, 77-114.

Dube, W. V. (1991). Computer software for stimulus control research with Macintosh computers. Experimental Analysis of human Behavior Bulletin, 9, 28-30.

Fernandes, J. C. (2002). Acústica e ruídos. Bauru, SP: Unesp.

Filgueiras, J. T. Q. (2011). Efeitos do treino discriminativo com resposta de seleção ou de tocar teclado sobre a leitura musical (Dissertação de Mestrado, Universidade de Brasília, Brasília, DF, Brasil).

Fiorentini, L., Arismendi, M., \& Yorio, A. A. (2012). Una revisión de las aplicaciones del paradigma de equivalencia de estímulos. International Journal of Psychology and Psychological Therapy, 12(2), 261-275.

Grey, J. M. (1977). Multidimensional perceptual scaling of musical timbres. Journal of Acoustical Society of America, 61(5), 1271-1277.

Hallam, S. (2010). The power of music: Its impact on the intellectual, social and personal development of children and young people. International Journal of Music Education, 28(3), 269-289.

Halpern, A. R., Zatorre, R. J., Bouffard, M., \& Johnson, J. A. (2004). Behavioral and neural correlates of perceived and imagined musical timbre. Neuropsychologia, 42, 1281-1292.

Hayes, L. J., Thompson, S., \& Hayes, S. C. (1989). Stimulus equivalence and rule following. Journal of the Experimental Analysis of behavior, 52, 275-291. 
Huber, E. R. (2010). Avaliação do ensino cumulativo de relações entre estímulos musicais sobre a formação de classes, o desempenho recombinativo e o tocar teclado (Dissertação de Mestrado, Universidade de Brasília, Brasília, DF, Brasil).

Madeira, I. E. C. (2012). Aprendizagem musical e manutenção de classes de estímulos equivalentes Dissertação de Mestrado, Universidade Federal do Espírito Santo, Espírito Santo, ES, Brasil).

Marozeau, J., Cheveigné, A., McAdams, S., \& Winsberg, S. (2003). The dependency of timbre on fundamental frequency. Journal of Acoustical Society of America, 114(5), 2946-2957.

McAdams, S., Winsberg, S., Donnadieu, S., de Soete, G., \& Krimphoff, J. (1995). Perceptual scaling of synthesized musical timbre: Common dimensions, specifities, and latent subject class. Psychology Research, 58, 177-192.

Med, B. (1996). Teoria da música (4a ed. rev. e ampl.). Brasília: MusiMed.

Pereira, E. S. (2012). Discriminação de diferença de frequência de sons e aprendizagem de leitura musical (Dissertação de Mestrado, Universidade de Brasília, Brasília, DF, Brasil).

Perez, W. F., \& de Rose, J. C. (2010). Recombinative Generalization: An exploratory study in musical reading. The Analysis of Verbal Behavior, 26, 51-55.

Reis, L. F. T. (2007). Ensino de discriminação de acordes baseado em treino de abstração e formação de classes de equivalência Dissertação de Mestrado, Universidade Federal de São Carlos, São Carlos, SP, Brasil).

Reis, T. S., de Souza, D. G., \& de Rose, J. C. (2009). Avaliação de um programa para o ensino de leitura e escrita. Estudos em Avaliação Educacional, 20, 425-450.

Schellenberg, E. G. (2004). Music lessons enhance IQ. Psychological Science, 15(8), 511-514.

Seinfeld, S., Figueroa, H., Ortiz-Gil, J., \& Sanchez-Vives, M. V. (2013). Effects of music learning and piano practice on cognitive function, mood and quality of life in older adults. Frontiers in Psychology, 4, 810.

Serejo, P., Hanna, E. S., de Souza, D. G., \& de Rose, J. C. C. (2007). Leitura e repertório recombinativo: Efeito da quantidade de treino e da composição dos estímulos. Revista Brasileira de Análise do Comportamento, 3(2), 191-215.

Sidman, M. (1971). Reading and auditory-visual equivalences. Journal of Speech and Hearing Research, 14, 5-13.

Sidman, M. (1994). Equivalence relations and behavior: A research story. Boston: Authors Cooperative.

Sidman, M. (2000). Equivalence relations and the reinforcement contingency. Journal of the Experimental Analysis of Behavior, 74, 127-146.

Sidman, M., \& Tailby, W. (1982). Conditional discrimination vs. matching to sample: An expansion of the testing paradigm. Journal of the Experimental Analysis of behavior, 37, $5-22$.

Smith, L. D., \& Williams, R. N. (1999). Children's artistic responses to musical intervals. American Journal of Psychology, 112, 383-410.

Sollberge, B., Rebe, R., \& Eckstein, D. (2003). Musical chords as affective priming context in word-evaluation task. Music Perception: An Interdisciplinary Journal, 20(3), 263-282.

Tommys, Y., \& Fazey, D. M. A. (1999). The acquisition of pitch element of music literacy skills by 3-4-year-old pre-school children: A comparison of two methods. Psychology of Music, $27,230-244$. 
Verghese, J., Lipton, R. B., Katz, M. J., Hall, C. B., Derby, C. A., Kuslansky, G., . . B Buschke, H. (2003). Leisure activities and the risk of dementia in the elderly. The New England Journal of Medicine, 348(25), 2508-2516.

Wessel, D. L. (1979). Timbre space as a musical control structure. Computer Music Journal, 3(2), 45-52.

Wilkinson, K. M., \& Mcllvane, J. W. (2001). Methods for studying symbolic behavior and category formation: contributions of stimulus equivalence research. Developmental Review, 21, 355-374.

Recebido: 27/10/2016

Última Revisão: 30/04/2017

Aceite final: 13/06/2017

\section{Sobre os autores:}

Raone Mateus Rodrigues: Mestrando do programa de Pós-Graduação em Psicologia: Cognição e Comportamento da Universidade Federal de Minas Gerais (UFMG). Instituto Nacional de Ciência e Tecnologia sobre Comportamento, Cognição e Ensino (INCT) e o Laboratório de Estudos do Comportamento, Cognição e Aprendizagem (LECCA). E-mail: raonemateusrodrigues@gmail.com.

Átila Moreira Cedro: Mestrando no Programa de Pós-Graduação em Psicologia da Universidade Federal de Minas Gerais (UFMG). Instituto Nacional de Ciência e Tecnologia sobre Comportamento, Cognição e Ensino (INCT) e o Laboratório de Estudos do Comportamento, Cognição e Aprendizagem (LECCA). E-mail: atila.cedro@gmail.com

Rebeca Mendes Fonseca: Discente do curso de Psicologia da Universidade Federal de Minas Gerais. E-mail: rabechebeca@gmail.com

Clarisse Vasconcelos Friedlaender: Discente do curso de Psicologia da Universidade Federal de Minas Gerais. E-mail: vf.clarisse@gmail.com

Pedro Henrique Ribeiro Torres: Discente do curso de Psicologia da Universidade Federal de Minas Gerais. E-mail: contatopedrotorres@gmail.com

Vinicius Figueiredo de Oliveira: Discente do curso de Psicologia da Universidade Federal de Minas Gerais. E-mail: viniciusfo96@gmail.com

Pedro Henrique Guimarães Couto: Discente do curso de Psicologia da Universidade Federal de Minas Gerais. E-mail: pedrohgcouto@gmail.com

Antonio Carlos Leme Junior: Docente do curso de graduação presencial em Música Licenciatura (Educação Musical) da Universidade Federal de São Carlos (UFSCAR). E-mail: antoniocarloslemejr@gmail.com

Edson Massayuki Huziwara: Docente do Programa de Pós-Graduação em Psicologia: Cognição e Comportamento da Universidade Federal de Minas Gerais (UFMG). Instituto Nacional de Ciência e Tecnologia sobre Comportamento, Cognição e Ensino (INCT) e o Laboratório de Estudos do Comportamento, Cognição e Aprendizagem (LECCA). E-mail: huziwara.edson@gmail.com 
